\title{
Energy efficiency tools for residential users
}

\author{
Emmanouil Zacharioudakis ${ }^{1, *}$, Helen C. Leligou ${ }^{1}$, Aikaterini Papadopoulou ${ }^{1}$ \\ ${ }^{1}$ Synelixis Solutions Ltd., Perissou 157, Nea Chalkidona, 14343, Athens, Greece, \{zacharioudakis, nleligou, \\ kpapadopoulou\}@synelixis.com
}

\begin{abstract}
Residential energy consumption reserves a significant portion of the total energy consumption in modern cities. The rates of construction of new buildings as well as the rates of renovation on existing ones are generally very low. At the same time, unlike centrally operated large commercial buildings, the installation of energy management systems is a rather expensive solution leaving residential users with limited means to improve their energy efficiency as results are not evident. Considering that to drive energy efficient behaviour, we have to first raise awareness, then provide evidence through measurements and then support further, more elaborate, energy efficiency actions, we capitalise on ICT as soft measures towards reaching hard goals. We propose a novel incremental solution starting from a rather simple mobile application exploiting the sensors available in our smartphones and tablets to proceed to more intelligent advice provisioning towards energy efficiency. We present its implementation architecture and discuss certain market-wise challenges to prove its potential.
\end{abstract}

\section{Introduction}

Energy consumed in the buildings' sector consists of residential and commercial end users and accounts for $20.1 \%$ of the total delivered energy consumed worldwide[1]. Buildings in the EU are responsible for $40 \%$ of primary energy consumption [2]. In households in particular it is $26.2 \%$ of total final energy consumption for EU28 [3]. By improving the energy efficiency of residential and public buildings, the EU's total energy consumption can be reduced by about $6 \%$ and lower $\mathrm{CO}_{2}$ emissions by about 5\%, [4], [5]. However, reducing energy use in buildings by introducing new technologies is very challenging; across Europe, the rates of construction of new buildings as well as the rates of renovation of existing ones are generally very low [6].

In order to achieve the energy reduction targets set for a sustainable future, affecting the behavioral characteristics of the citizens' interaction within the buildings where they live and work, will have a more tangible impact [6]. Human beings need knowledge and motivation to change and Information and Communication Technologies (ICT) can assist in both providing knowledge and communicating the motivation. Raising awareness of the importance of efficient use of energy is a major target in many countries as this is the most long-lasting measure towards energy consumption reduction. ICT is a lowcost solution in shaping behaviours and achieving energy efficiency. This approach is currently pursued by different initiatives among which the EU-GAIA project focuses on a particular target group as essential for designing a successful behavioral-change strategy [7]. Observing that educational buildings constitute $17 \%$ of the non-residential building stock in the EU [8], GAIA focuses on the educational community, aiming to instill behavioral change towards energy efficiency and sustainability through an educational approach, combined with a set of applications.

Targeting energy efficiency in residential setting can yield significant results because a) motivating people to embrace energy-efficient habits has sustainable results on energy consumption, as these people are highly unlikely to abolish their habits in the future as well as in other environments and b) people tend to imitate behaviours which leads to a multiplicative effect. Research interviews conducted [9] made clear that once learned, energy conservation insights can be applied everywhere.

To shape the behaviour of EU residents, a set of ICT tools can be exploited. First, knowledge needs to be acquainted about the importance of behaving in an energy efficient manner and actions that can lead in significant energy reductions. This can be achieved through relevant games and/or quizzes prompting the reader to visit sites with appropriate information, towards achieving the goal of spreading knowledge in an enjoyable manner. Second, practical advice on how to reduce energy consumption in specific real-life circumstances needs to be provided. Again, ICT can accomplish this e.g. through mobile phone applications, for specific real-life situations, either manually entered by the user or sensed by the device itself. Third, advice

\footnotetext{
Corresponding author: zacharioudakis@,synelixis.com
} 
taking into account real energy consumption information is highly valuable and can result in significant energy savings. Psychologists agree that you have to measure before you act, since measurements provide quantifiable information about the benefits of acting green. This can be achieved either through participatory sensing or through the installation of measurement infrastructure (e.g. power meters, temperature, luminosity sensors) whose cost can range from few hundreds of euros (2017) to few thousands, depending on the measurement points, accuracy and intelligence of the accompanying software.

The rest of the paper is organized as follows: in section 2, we provide a short introduction to participatory sensing and on currently available solutions for residential use. In section 3, we present a set of applications towards shaping behaviours and assisting residential users in being energy efficient. In section 4, we present the implementation architecture of the application set, in section 5 we present the challenges, and finally in section 6 we conclude discussing the potential of such an application set.

\section{Currently available solutions -State- of-the-art}

\subsection{Participatory sensing}

Participatory sensing is the concept of communities (or other groups of people) contributing sensory information to form a body of knowledge. The ideas behind participatory sensing is rather than design and build fancy (and expensive) occupancy sensors, we instead rely on the building's occupants to participate in the sensing process. Apart from collecting valuable information, having the occupants insert information about temperature, luminosity and power consumption helps them realise how their behaviour impacts energy consumption and its interplay with the experienced comfort. Additionally, participatory sensing has been shown to create wisdom in many different sectors from weather, urban mobility, congestion as well as any other sensory information that collectively forms knowledge.

The idea is to collect measurements through a mobile device or a laptop/desktop computer in a central repository and process/visualise the readings so as to support intelligent decision making. In a building context, a simple smartphone (android/iOS/SMS-based) application where building occupants can provide realtime feedback on room-level building comfort could be considered as participatory sensing information for collecting comfort level information. The app would prompt the user to enter the building and room number and then allow them to rate the comfort of the room using a (rather limited) thermal sensation scale, e.g. from $(-3)$ very cold to $(+3)$ very hot $(0$ means comfortable; this scale is the "industry standard" for measuring thermal comfort). These reports would then be collected in a central database for analysis. A web-based interface would then be created to visualize the data. One visualization could be a dynamic overlay of recent thermal comfort reports on the building floorplan; other visualizations could show the historical comfort of different rooms at different times of the day while more advanced analyses are also possible by collecting additional data and performing more sophisticated processing.

\subsection{ICT applications for energy efficiency}

Energy efficiency relevant applications span diverse disciplines and may have different objectives all aiming at contributing towards the major goal of energy consumption reduction. Among them are energy consumption simulators for building/districts/countries, energy management systems for buildings (suitable mainly for big buildings where dedicated personnel for building management exists), smart home applications enabling remote control of devices towards enhancing comfort and reducing energy consumption, e.t.c. Up to now, solutions for energy management and thus efficiency in buildings rely on sensing and actuating infrastructures which are rather expensive for residential users who however, could create a significant impact on energy consumption if they had the means to collect data, receive recommendations and act upon the provided input.

Example applications for participatory or user-based sensing are Meter Readings and MobilizingCS. Meter Readings is a tool that makes it easy to regularly monitor the energy usage of the household. The user is allowed to add the types of meters to monitor, and to select an interval. Then the app will notify the user when it is time to read the meters. Based on the readings entered, it is possible to get a graphical overview of the monthly usage as well as total average and usage per day. MobilizingCS is a data collection tool; it provides mobile-based data capture through inquiry-based surveys and temporarily triggered reminders. All captured data are automatically timestamped, geocoded and uploaded to a centralized MobilizingCS server for analysis and visualization. The current application is the first step towards realizing the vision of a planetary nervous system. In this version, users are able to collect real-time data from their mobile phone with a user-controlled frequency. The data are stored in the phone and can also be pushed to a remote location that users self-determine. The sensor data can be visualized in real-time. Moreover, the application provides two levels of privacy control to users in the data collection process: (i) privacy by determining which sensor data are locally stored in the phone and (ii) privacy by determining which sensor data are anonymized and remotely shared.

ICT-based solutions relevant to energy management systems for buildings include systems that come with sensing and actuating infrastructures along with software capable of visualising the readings, of providing recommendations and of supporting building manager decision making and execution in a user friendly manner. Such systems have been developed by numerous industrial stakeholders like $\mathrm{ABB}$ and Honeywell and include BuildingIQ [10], Decathlon, EnergyCAP [11], eSightenergy [12] Rockwell 
Automation [13], Energy Lens [14], and many others. For example, BuildingIQ reduces Heating, ventilation and air conditioning (HVAC) energy costs in commercial buildings, works with a Building Management System (BMS) for actuating purposes, continuously monitors inputs including weather, occupancy, energy prices and demand response events, makes small changes at the air handler unit (AHU) level that result in large HVAC energy savings, without impacting occupant comfort, improves a building's energy performance and usage without incurring CapEx expenses, reduces a building's carbon footprint and improving its sustainability score by up to 20 LEED points or a full NABERS point, enables a building to be Smart Grid-ready without requiring an upgrade or custom engineering.

\section{A scalable solution for residential users}

Our aim is to exploit state-of-the-art technologies to build a scalable solution that can guide residential users behave in a more energy efficient manner. To do so, they would be supported to track and control their home's energy consumption and to make efficiency aware decisions.

\subsection{Raising awareness}

As the first step in behavioural change is to raise awareness, the elementary component of the proposed solution consists of a smartphone application which focuses on luminosity and temperature conditions and user guidance to adjust them: saving energy while preserving their comfort. With respect to luminosity, it exploits the currently available in all smartphone devices luminosity sensors, captures the luminosity value, displays it to the user and prompts them to choose among a set of activities. Depending on the selected activity and combining it with the current time, the application notifies the user if the luminosity is adequate for their activity, if more than the suitable value for the activity it provides a message informing them about potential savings. It would tell them that regulating the luminosity could save them a certain amount of energy (and possibly, relevant cost, if inserted at the application initialisation time) and could also reduce the $\mathrm{CO}_{2}$ footprint by a certain amount. With respect to temperature, unfortunately the temperature sensor existing in mobile devices cannot be exploited because it is placed close to the device's processor aiming at sensing its temperature and avoid overheating. Thus, the user is prompted to enter the temperature values on their own. Once entered, they are, similarly with the previous case, provided with recommendations about taking action to reduce the energy consumed to reach the desired indoor temperature. For example, they are notified about the target values ensuring comfort by widely accepted standards, they are informed about the energy expenses associated with every degree in the temperature and how this correlates with the external temperature as well as about easy to take actions.

The proposed solution provides recommendations based on the wide literature and statistics available today, while additional recommendation or exchange of ideas could take place through social networking among all users of the specific application.

\subsection{Systematic monitoring and measurement}

On top of the previous application, a web-based mobile application that allows for manual uploads of home energy sources values-electricity, oil, natural gas- can assist residential users in closer monitoring of the energy consumption in their houses. Through this application, the user is prompted to insert power consumption values entering readings from the power meter either through a dedicated user interface or by uploading power consumption information through a structured file e.g. an excel file. The readings are uploaded to a cloud infrastructure where they are processed and visualised. Apart from the visualisation of the reading, the application provides additional information such as statistics about average consumption of other residential users and comparison to historical reading from the same house, if available. This way the residential user can a) observe peaks of energy consumption and take action if not justified by their activities, b) observe the base energy consumption and identify which devices consume energy even if no other load is active and c) study whether specific loads can be shifted in time to match demand-response programs put in place by the power provider. Analysis of historical data is provided via attractive diagrams and important metrics such as mean values, peak and base loads depicted in a daily, weekly or monthly basis according to the user preference.

\subsection{Semi-automated Energy Management}

The next step is a) more elaborate monitoring and b) user guidance based on specific rules, assuming the absence of actuators. For more elaborate monitoring, the installation of sensing devices (power meters and possibly temperature sensors) should be placed in the building tracking energy consumption, temperature and humidity levels in order to automate the procedure. Two options arise here: either the infrastructure provider delivers software capable of extracting the reading in some structured file, in which case, this can be imported in the above mentioned participatory sensing part of the solution; or enable the integration of the sensors with the provided cloud infrastructure, assuming this will have an open Application Programming Interface implemented for this reason. This way, more elaborate monitoring can be achieved guiding more elaborate decisions. It is worth stressing that sensing equipment can be nowadays installed by the users themselves, i.e. it does not require the intervention of specialised personnel. This is an important element as one can buy a one-phase power meter and use it to monitor different power lines each day/week/month. Additionally, in certain markets, it is 
possible to rent equipment which allows for elaborate monitoring for a specific time span. According to the observation during this time span, specific rules can be defined, upon which the user is notified and instructed to act appropriately.

With respect to actions, assuming that no actuators are in place, the application allows the user to define rules upon which they are notified. Alerts notify the user when rules are triggered via text or email allowing them to take appropriate action. A social networking feature for users to compare similar buildings is possible while the application can also be available through desktop computers. For example, "If the temperature exceeds 22 degrees Celsius, send an SMS". While this seems to be over simplistic, there are still buildings where no thermostat in the rooms exists and the thermostat is only installed where the HVAC central system is installed. Additionally, there are many cases where the building thermal profile has radically changed from the time the initial study had been performed, leading to an apartment being over-heated while other remain at low temperatures. A flexible notification generation engine enabling the user to specify rules at will, can become a powerful tool since all major energy management systems are in essence executing specific rules.

While the main motivation can be considered the combination of environment sustainability with the reduction of energy bills, additional motivation can be provided by gamification/rewarding schemes which can be implemented on top of the previous application components and potentially combined with social networking flavours. This can be considered as a separate component which compares current with past user behaviour and energy bills and creates a hall of fame among users so that more energy efficient users become known and they can gain popularity.

\section{The implementation architecture}

The proposed application set exploits the sensing elements of the mobile device, sensing devices installed at home, visualisation and web technologies and the abundant processing and storage facilities offered at cloud infrastructures. As shown in Fig. 1, so as to raise awareness a mobile application is proposed which can be executed on the mobile phone while, by communicating with the cloud, the repertoire of the recommendations provided can be continuously enriched and updated. To be able to read the sensors existing in the mobile devices, native apps have to be developed for Android and $\mathrm{iOS}$ devices, as these are the ones exhibiting high penetration to the populations.

To support participatory sensing in the targeted environment, a web-based application can be used (which can be executed both at desktop/laptop computers and at mobile devices, e.g. smartphones and

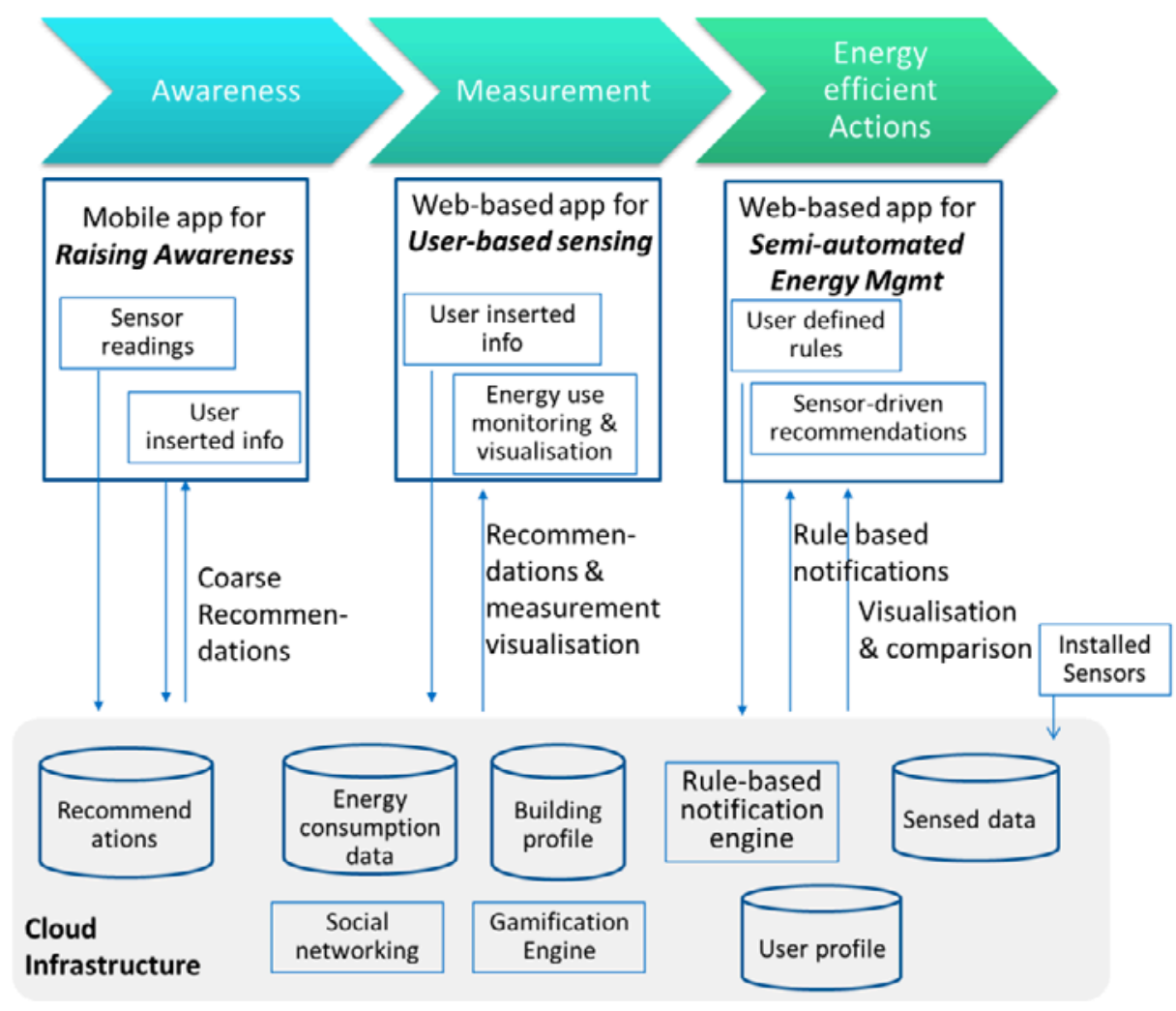

Fig.1. The implementation architecture of the application set realising the concept of raising awareness, measure and act in a efficient manner

tablets). Through a user friendly interface this application allows for a) inserting user-read data from power meters and/or temperature/humidity sensors b) inserting reading from power utilities or exported from sensing devices and c) inserting building specific information. Specifically, to create enhanced user 
experience, the user interface can be based on HTML5, CSS3 and on the materialize CSS (material design) framework. Also AngularJS framework version 1.5 can be used for the further necessities.

The user is furthermore able to insert building specific information including building/apartment ID, number of floors/areas in the building, number of individual rooms (e.g. classrooms) in the floor/area, geographic location, surface $\left(\mathrm{m}^{2}\right)$ per area, number of inhabitants per house/area, age of the building, whether there was intervention /Date of intervention per building, orientation, energy types available (electricity, gas, etc.).

With respect to the energy consumption, the user is allowed to insert yearly consumption per energy type, yearly energy cost and footprint.

The user will receive valuable information including:

-Visualised building performance information allowing for comparing the performance among days/months/years as well as for comparing the performance of the currently monitored house/apartment with others of the same typology. By typology we refer to houses/apartments of the same/similar geographical location (as it is not objective to compare an apartment in Athens with an apartment in Sweden), of the same age/thermal characteristics which strongly depend on the material used for its construction and of similar use (residential buildings should be compared to residential buildings). As the number of inhabitants, strongly affects the consumed energy, in the analytics the capability to present energy consumption per inhabitant will be offered.

-Receive suggestions for energy savings. These can range from suggestions for long-term actions and interventions (such as "you consume too much for heating, consider changing the central heating to another method") to suggestions like "electricity consumption is higher than other days; is it justifiable? Consider making out the most of natural light".

The visualisation itself is expected to provide adequate insight to the users to seek ways to reduce the peaks as well as average energy consumption values. For example, as shown in Fig. 2, it is evident that in the period visualised at the top graph, the peak energy is higher even through the average of the two graphs may be quite close one another.

Such comparisons may lead to valuable observation suggesting devices that have highly fluctuating energy demand patterns which may drive the user to activate these devices in time periods that the power utility offers more attractive tariffs. As regards the intelligence of the suggestions, this depends on the collected readings as well as on the implementation of a relevant recommendation engine.

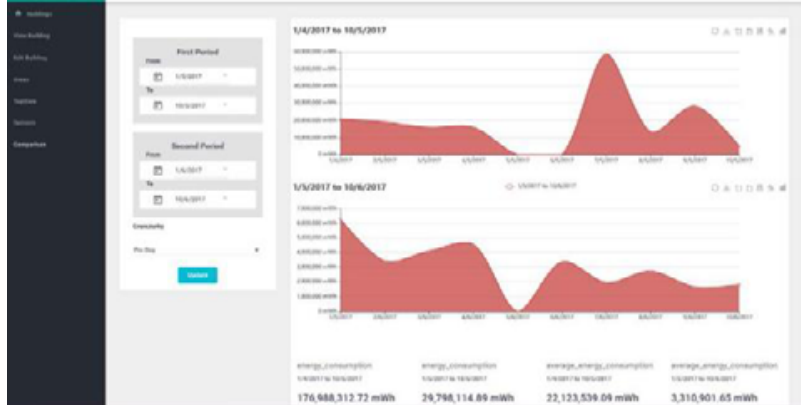

Fig. 2. Comparison of measurements in two different time periods

Finally, the 3rd step incorporates reading from sensing devices. As Internet of Things (IoT) technologies invade our everyday lives, interconnected sensing devices become a commodity and interfaces are getting standardised. Each installation may consist of a multitude of IoT nodes, which communicate with a cloud infrastructure via a gateway device. The IoT nodes include multiple sensing devices, while the gateway nodes coordinate communication and enable interaction with cloud-based services and other Internet-connected devices. All devices use a wireless interface to communicate locally, while gateways use wired connectivity to report measurements and receive commands or configuration updates.

The overall design pattern for the installation of IoT infrastructure in the considered buildings is as follows:

-IoT nodes installed to monitor the power consumption of the building/house as a whole, or specific areas/sectors (e.g. individual power lines).

-IoT nodes installed in rooms to monitor a set of environmental parameters such as temperature, humidity, activity and noise levels (see Fig. 1).

- One or a set of IoT gateway nodes installed in central points of the house/building to bridge the IoT nodes that communicate using IEEE 802.15.4 with the Internet.

Assuming that a) the IoT gateways communicate directly with some cloud infrastructure which can be accessed by our applications or b) at least the relevant readings can be exported and re-inserted in our cloud infrastructure, continuous monitoring of energy consumption and environmental parameters is possible. If these data are available in near real time, then the user can define rules upon which they receive a notification triggering their actions. Default rules will be offered by the application itself although greater savings can be achieved if the user takes the advice of an expert and sets accordingly their own rules. The same notification engine residing in the cloud can be exploited to generate alerts, e.g. when inhabitants are away and temperature values exceed a predefine threshold or water consumption is not zero. It needs not pointing out that comparison with other similar residences is still possible and all previously defined suggestions are still enacted. An example of the information accompanying an alert is shown in Fig. 3. 


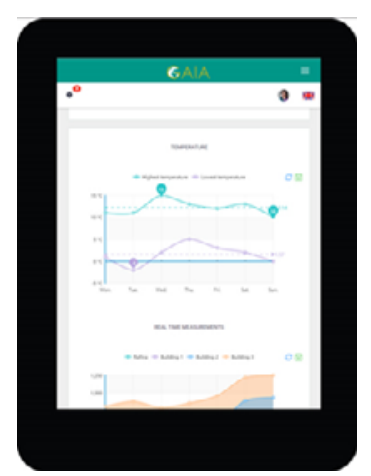

Fig. 3. Information provided together with an alert about detected consumption anomalies

\section{Challenges}

As the aforementioned applications capitalise on stateof-the-art technologies, the main challenges in their implementation and mass deployment stems from business relevant matters. The first application outlined above is a mobile app which can either be offered for free to trigger the audience's interest or advertisement of Energy Services COmpanys (ESCOs) can pay off its deployment. ESCOs are entities that primarily invest in building interventions and are paid off by the energy savings achieved. In the second step, for users to be willing to pay for using it or downloading it, it is necessary to enrich the recommendation engine with sophisticated and high-value recommendations which mandates cooperation with building energy management experts. Finally, in the third step, it is important to ensure integration of a wide set of available in the market sensing devices in the infrastructure used by the presented applications. This mandates the implementation of so-called wrappers for popular sensing device interfaces.

\section{Conclusions}

Residential buildings consume an important percentage of total energy consumption in modern cities. Towards reducing the consumed energy and increasing the efficiency of energy use, first, awareness about energy efficiency should be raised, then, this should be supported by measurements to provide adequate evidence to users and finally, suggestions at all timescales, (from intervention to real-time actions) should be provided to the users.

In this paper, we have proposed an incremental solution that can be used by residential users to achieve high energy efficiency and reduce energy bills obviating the need for installing expensive energy management systems. The proposed solution capitalises on state-ofthe-art technology to ensure low cost, while initial business aspects have been explored to check business viability.

\section{Acknowledgment}

The work presented in this paper is partially funded by the Green Awareness in Action (GAIA) project funded by the European Commission under the H2020-EE2015-2-RIA call and contract number 696029.

\section{References}

1. EIA, International Energy Outlook 2016, Chapter 6 Building Energy Consumption (2016) https://www.eia.gov/forecasts/ieo/buildings.cfm

2. European Commission, Energy, Buildings, https://ec.europa.eu/energy/en/topics/energyefficiency/buildings

3. F. Christian, Statistics on Energy Consumption in Households (2014)

4. https://ec.europa.eu/energy/en/topics/energyfficiency/buildings

5. SIEMENS, Building Automation Impact on Energy Efficiency, Application per EN 15232 eu.bac product certification

6. Buildings Performance Institute Europe, Europe's buildings under the microscope: A country-bycountry review of the energy performance of buildings, Technical report, (2011)

7. J. Stromback, C. Dromacque, M. H. Yassin, The potential of smart meter enabled programs to increase energy and systems efficiency: a mass pilot comparison, Technical report, VaasaETT Global Energy Think Tank, Funded by European Smart Metering Industry Group (2013)

8. http://gaia-project.eu/index.php/en/homepage-3/

9. K. Crosby, A. B. Metzger, Powering down: A toolkit for behavior-based energy conservation in $k-12$ schools, Technical report, U.S. Green Building Council (2012)

10. https://www.buildingiq.com/solutions

11. http://www.energycap.com

12. http://www.esightenergy.com/uk/software

13. http://www.rockwellautomation.com

14. http://www.energylens.com/ 